\title{
Tunable moire spinons in magnetically encapsulated twisted van der Waals quantum spin liquids
}

\author{
Guangze Chen $(1)$ and J. L. Lado $\odot$ \\ Department of Applied Physics, Aalto University, 02150 Espoo, Finland
}

(Received 19 February 2021; revised 5 July 2021; accepted 31 August 2021; published 24 September 2021)

\begin{abstract}
Quantum spin-liquid van der Waals magnets such as $\mathrm{TaS}_{2}, \mathrm{TaSe}_{2}$, and $\mathrm{RuCl}_{3}$ provide a natural platform to explore new exotic phenomena associated with spinon physics, whose properties can be controlled by exchange proximity with ferromagnetic insulators such as $\mathrm{CrBr}_{3}$. Here we put forward a twisted van der Waals heterostructure based on a quantum spin-liquid bilayer encapsulated between ferromagnetic insulators. We demonstrate the emergence of spinon flat bands and topological spinon states in such heterostructure, where the emergence of a topological gap is driven by the twist. We further show that the spinon band structure can be controlled via exchange proximity effect to the ferromagnetic leads. We finally show how by combining small magnetic fields with tunneling spectroscopy, magnetically encapsulated heterostructures provide a way of characterizing the nature of the quantum spin-liquid state. Our results put forward twisted quantum spin-liquid bilayers as potential platforms for exotic moire spinon phenomena, demonstrating the versatility of magnetic van der Waals heterostructures.
\end{abstract}

DOI: 10.1103/PhysRevResearch.3.033276

\section{INTRODUCTION}

Magnetic van der Waals materials have risen as a highly versatile family of compounds in the two-dimensional realm [1-4]. These materials attracted much research interest as their two-dimensional nature provides a platform to electrically control magnetism [5-8], design magnetic tunnel junctions [9-12], topological superconductivity [13,14], and exploit magnetism in generic van der Waals heterostructures [15]. Remarkably, specific magnetic van der Waals materials such as $\mathrm{TaS}_{2}, \mathrm{TaSe}_{2}$, and $\mathrm{RuCl}_{3}$ provide a realization of an exotic phase of matter, quantum spin-liquid (QSL) states [16-19].

In contrast with conventional magnets, QSLs appear in magnetic systems featuring strong degrees of frustration, and are characterized by a quantum disordered ground state [20-24]. Interest in quantum-spin liquids has been fueled by their potential emergent Majorana physics [25] and their potential relation with high-temperature superconductivity [26,27]. A variety of materials have been proposed as QSL candidates [28-40], yet it remains a remarkable challenge to unveil the nature of QSLs and to experimentally identify them $[41,42]$. Interestingly, magnetic van der Waals materials offer new directions for the engineering and detection of QSL, by exploiting the large flexibility of stacking and twisting of moiré systems.

Stacking van der Waals heterostructures yields electronic structures sensitive to the relative twisting between different layers [43,44]. A paradigmatic example of these

Published by the American Physical Society under the terms of the Creative Commons Attribution 4.0 International license. Further distribution of this work must maintain attribution to the author(s) and the published article's title, journal citation, and DOI. phenomena is twisted bilayer graphene, where the emergence of flat bands has led to a variety of unconventional many-body states [45-49]. Interestingly, twist engineering generically provides a platform for correlated phases with electrical tunability [50-52] and topologically nontrivial electronic structures [53-58]. The versatility offered by stacked van der Waals heterostructures motivates the search for analogous phenomena in the realm of van der Waals magnets [59-61] that can lead to novel spinon phenomena in moiré quantum spin liquids. Ultimately, the moiré quantum spin liquids would allow the detection of spinons, which remains a much bigger challenge than detecting magnon counterparts in ordered van der Waals magnets [9].

In this work, we put forward twist engineering in QSL van der Waals heterostructures as a powerful knob to control spinon physics. We show that twist engineering creates spinon flat bands at a specific twisting angle, with a topological gap opening leading to in-gap spinon edge modes. We show that the spinon spectra can be tuned by means of encapsulation between van der Waals magnets, leading to dramatic changes in their low energy spectra. Finally, we discuss how this exchange bias tuning provides a spectroscopic electrical method to characterize QSL states. Our results put forward magnetic van der Waals heterostructures formed by ferromagnets and QSLs as a tunable platform to explore and probe spinon phenomena in moiré systems.

Our paper is organized as follows. In Sec. II, we show the spinon spectra in a twisted bilayer QSL structure, where spinon flat bands and a topological gap opening emerges. In Sec. III, we show that magnetic encapsulation allows tuning the spinon spectra in the twisted QSL, that can be eventually probed with inelastic spectroscopy. In Sec. IV, we elaborate on specific quantitative aspects of our proposal relevant for experiments. Finally in Sec. V, we summarize our conclusions. 


\section{SPINON SPECTRA IN TWISTED BILAYER QUANTUM SPIN LIQUID}

For the sake of concreteness, in the following, we focus on a specific gapless quantum spin liquid state on the triangular lattice, structurally analogous to the one proposed for $\mathrm{TaS}_{2}$. For this sake, let us first briefly review the physics of a single-layer QSL. We start with a Heisenberg model on a triangular lattice of the form $\mathcal{H}_{0}=\sum_{i, j} J_{i j}^{\mu \nu} S_{i}^{\mu} S_{j}^{v}$ where $J_{i j}^{\mu v}$ is the exchange coupling between spin components $\mu, v$ on sites $i, j$. The previous model is known to have a rich phase diagram and in particular it supports QSL states such as those realized in $\mathrm{TaS}_{2}[16,62,63]$, $\mathrm{TaSe}_{2}[18]$, and $\mathrm{NaYbO}_{2}$ $[39,40]$. We now focus on the regime of the model yielding a QSL state with a linear density of states (DOS), and in particular the $U(1)$ Dirac spin liquid $\pi$-flux model $[64,65]$. This state can be captured by performing a parton transformation of the form $\mathbf{S}=\frac{1}{2} f_{\alpha}^{\dagger} \sigma_{\alpha \beta} f_{\beta}$, with $f_{\alpha}^{\dagger}$ and $f_{\alpha}$ fermionic spinon operators and $\sigma_{\alpha \beta}$ the spin Pauli matrices. With the previous replacement, the Heisenberg model can be solved at the spinon mean-field level, yielding a single-particle spinon Hamiltonian of the form $H_{0}=t \sum_{\langle i, j\rangle} \chi_{i j} f_{i}^{\dagger} f_{j}$, where $\chi_{i j}$ and $t$ are mean-field parameters. The $\pi$-flux model is defined by taking the mean-field solution $\chi_{i j}$ hosting an associated staggered 0 and $\pi$ fluxes in neighboring triangles. We apply the gauge with real hoppings $\chi_{i j}= \pm 1$ such that the system has time-reversal symmetry. Under this gauge, the model has two Dirac cones in the first Brillouin zone located at time-reversal invariant momenta [66].

We move on to consider a twisted bilayer QSL as sketched in Fig. 1(a). We start from the parent Heisenberg Hamiltonian for the twisted bilayer, that takes the form

$$
\mathcal{H}=\sum_{l, i, j} J_{\|, i j}^{\mu \nu} S_{i, l}^{\mu} S_{j, l}^{\nu}+\sum_{i, j} J_{\perp, i j}^{\mu \nu} S_{i, 1}^{\mu} S_{j, 2}^{\nu},
$$

where $l$ labels the two layers, and $J_{\|, i j}^{\mu \nu}$ and $J_{\perp, i j}^{\mu \nu}$ denote intra- and interlayer spin exchange, respectively. In the regime $J_{\|, i j}^{\mu \nu} \gg J_{\perp, i j}^{\mu \nu}$, the ground state of the system will consist of two coupled $U(1)$ QSL states. Therefore, we take as the meanfield solution for each layer the spinon $\pi$-flux model, with an effective interlayer spinon coupling from interlayer spin exchange:

$$
H=t \sum_{l,\langle i, j\rangle} \chi_{l, i j} f_{i, l}^{\dagger} f_{j, l}+\sum_{i, j} t_{\perp, i j}\left(f_{i, 1}^{\dagger} f_{j, 2}+\text { H.c. }\right),
$$

where $t, \chi_{l, i j}$, and $t_{\perp, i j}$ are mean-field parameters that can be derived analogously from a mean-field replacement in Eq. (1). The mean-field parameter $t_{\perp, i j}$ will depend on the relative distance between sites $i$ and $j$, inherited from the parent Heisenberg coupling $J_{\perp, i j}^{\mu \nu}$ in Eq. (1). We take a functional form for the interlayer coupling as [67] $t_{\perp, i j}=$ $t_{\perp, 0} \frac{d^{2}}{r_{i j}^{2}} e^{-\lambda\left(r_{i j}-d\right)}$, where $d$ is the interlayer distance, $r_{i j}$ is the distance between sites $i$ and $j, \lambda$ is the parameter that controls the decay of the interlayer coupling, and $t_{\perp, 0}$ is the largest possible interlayer coupling realized at $r_{i j}=d$. In the following we take $t_{\perp, 0}=0.36 t, \lambda=10 / a$, and $d=a$, where $a$ is the lattice constant of the triangular lattice. From the computational point of view, we will use the twist scaling relation for computational convenience [68-70], and we compute the

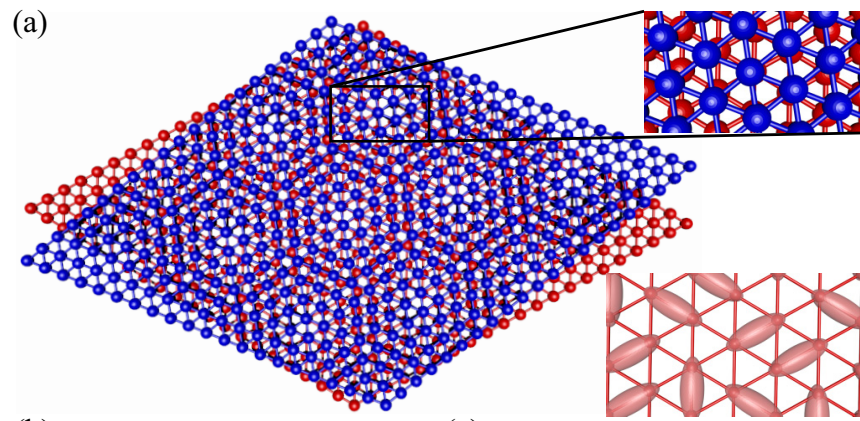

(b)

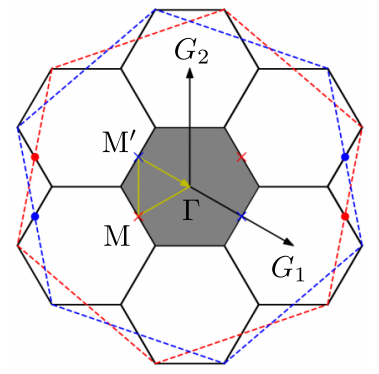

(c)

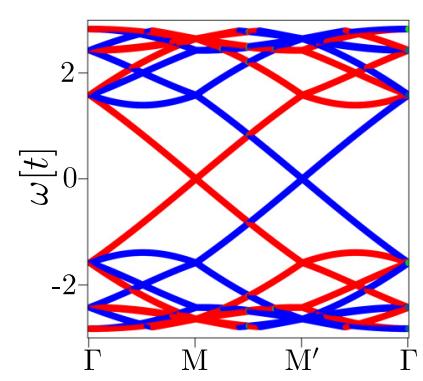

FIG. 1. (a) Sketch of a twisted bilayer Dirac QSL on a triangular lattice. (b) The moiré Brillouin zone of the twisted QSL. The dashed red and blue hexagons represent the Brillouin zone of the bottom and top layers. The red/blue circles (crosses) denote the location of the Dirac points in the original (folded) Brillouin zone (grey area). (c) Spinon band structure of the twisted Dirac QSL in the decoupled limit for $\theta=22^{\circ}$, showing the two sets of decoupled bands in the top (blue) and bottom (red) layers.

valley expectation $\left\langle\mathcal{V}_{z}\right\rangle= \pm 1$ by means of the valley operator [51,71-74].

The $\pi$-flux hoppings $\chi_{l, i j}$ are subject to a $U(1)$ degree of freedom for each layer, respectively. However, the gauge difference between the two layers determines the relative position of Dirac cones of the two layers in reciprocal space [75]. As a result, the momentum difference between Dirac cones of the two layers, $\Delta \mathbf{k}$, can be either large or small. When $|\Delta \mathbf{k}| \gg|1 / \mathbf{R}|$, where $\mathbf{R}$ is the periodicity of $t_{\perp, i j}$ in real space, the Dirac cones are almost decoupled. In such case, the impact of $t_{\perp, i j}$ is small on low energy physics, keeping the two layers effectively decoupled. In contrast, when $|\Delta \mathbf{k}| \ll|1 / \mathbf{R}|$, $t_{\perp, i j}$ leads to significant coupling between the Dirac cones of different layers. From the energetic point of view, this gauge configuration couples the two layers and therefore will lower the many-body energy through spinon hybridization. With this gauge choice, the moiré Brillouin zone is shown in Fig. 1(b), with two Dirac cones at time-reversal invariant momenta $M$ and $M^{\prime}$. The spinon dispersion in the decoupled limit for twisting angle $\theta \approx 22^{\circ}$ is shown in Fig. 1(c), where the two spinon Dirac cones of the two decoupled layers are observed.

Let us now move on to the case in which the two QSLs are coupled through the interlayer exchange coupling. In this situation, we find that the interlayer coupling leads to a gap opening in the Dirac cones of the bilayer QSL upon twisting. This gap opening stemming from the twist is similar to the case of twisted double bilayer graphene [53] [Figs. 2(a)-2(c)], and stems from the broken $C_{2 z} T$ symmetry [76,77] of the effective model. Besides the gap opening, we also observe the 
(a)

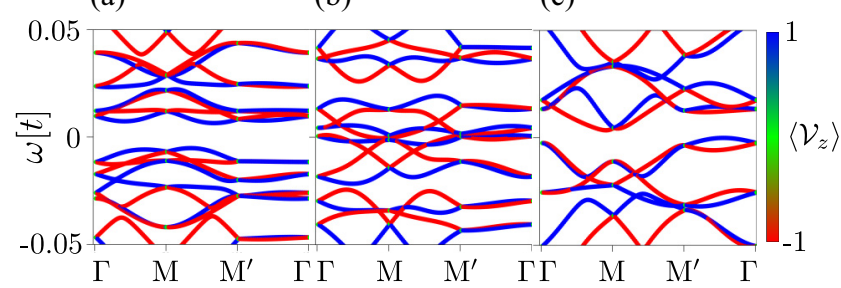

(d)

(e)
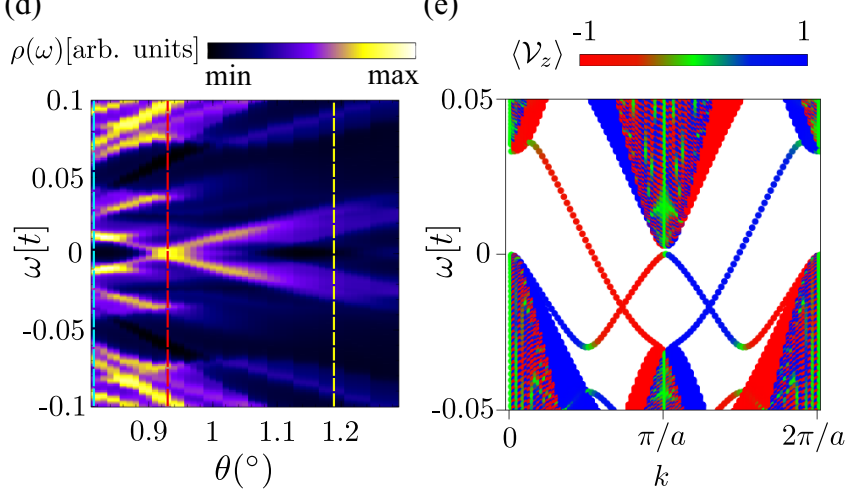

FIG. 2. (a)-(c) Spinon band structure of the twisted Dirac QSL at different twisting angles $\theta=0.81^{\circ}, 0.93^{\circ}, 1.20^{\circ}$ [dashed lines in panel (d)], respectively. (d) Spinon DOS $\rho(\omega)$ of the twisted Dirac QSL near the flat band twisting angle $\theta=0.93^{\circ}$. (e) Spinon band structure of a twisted QSL nanoribbon at $\theta=3.5^{\circ}$.

emergence of spinon flat bands at a specific fine tuned twisting angle $\theta /\left(t_{\perp} / t\right) \approx 2.6^{\circ}$, which for $t_{\perp}=0.36 t$ appears at $\theta=0.93^{\circ}$ [Fig. 2(d)], similar to other twisted Dirac materials $[43,44]$.

Interestingly, the emergence of a gap opening driven by the twist has been shown to give rise to topological states in van der Waals heterostructures based on graphene [78-80]. In particular, we find that the gap opening in the bilayer QSL has an associated valley Chern number of 2, giving rise to two counterpropagating channels at each edge with opposite valley polarization [81-85]. The previous phenomenology can be explicitly demonstrated by computing the spinon band structure of a twisted QSL nanoribbon at twisting angle $\theta=$ $3.5^{\circ}$ [Fig. 2(e)]. In particular, it is clearly seen the emergence of in-gap valley-polarized topological edge modes, associated with the topological valley Hall quantum spin-liquid state. We note that the topological edge modes are protected by the approximate valley charge conservation, and therefore perturbations giving rise to strong intervalley scattering can lead to intervalley mixing between topological spinon edge states [73,74,84,86,87].

\section{TUNING SPINON SPECTRA WITH MAGNETIC ENCAPSULATION}

After considering the emergent spinon spectra driven by the twist between the two QSL layers, we now move on to show how such emergent moiré bands can be controlled by a magnetic encapsulation. From the material science point of view, in the following we will consider that the QSL bilayer is sandwiched between two ferromagnetic insulators, for which (a)

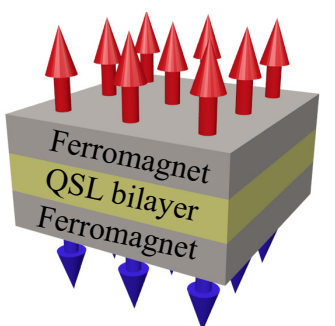

(c)

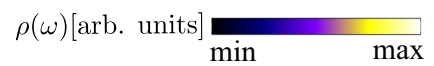

(d)

(b)
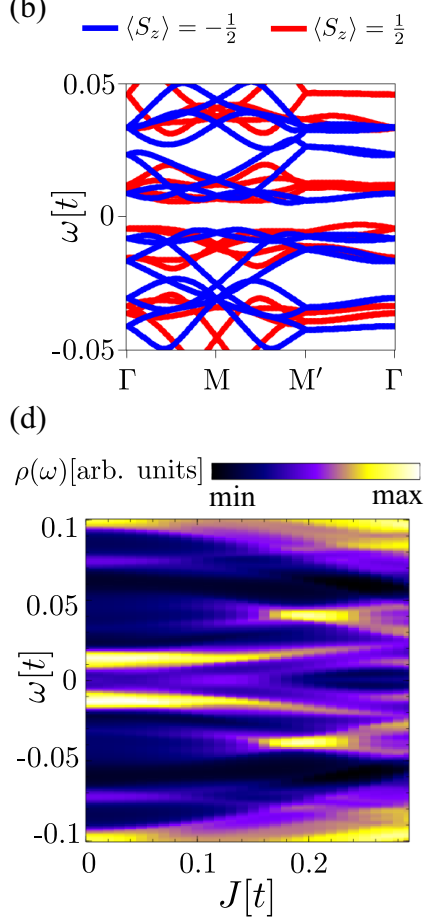

FIG. 3. (a) Sketch of the twisted bilayer Dirac QSL encapsulated by ferromagnets. (b) Spinon band structure of the twisted Dirac QSL at $\theta=0.93^{\circ}$ and interlayer spinon bias $J=0.1 t$. (c),(d) Spinon DOS of the twisted bilayer Dirac QSL at (c) $\theta=0.93^{\circ}$ and (d) $\theta=1.05^{\circ}$ with different interlayer spinon bias $J$.

both $\mathrm{CrBr}_{3}[9,88]$ and $\mathrm{CrCl}_{3}$ would be suitable candidates. The top and bottom ferromagnets are expected to be antiferromagnetically aligned through a superexchange mechanism [89], as shown in Fig. 3(a). To study the impact on the QSL state, we now integrate out the degrees of freedom of the ferromagnet, and consider their impact on the QSL Hamiltonian. The magnetic encapsulation yields an exchange proximity term in the Hamiltonian of the QSL bilayer, analogous to the exchange terms proposed for other van der Waals materials proximized to ferromagnets [51,90-93],

$$
\mathcal{H}^{\prime}=\mathcal{H}+\sum_{i, \mu} \mathcal{J}_{\perp} S_{i, 1}^{\mu} \mathcal{M}_{1}^{\mu}+\sum_{i, \mu} \mathcal{J}_{\perp} S_{i, 2}^{\mu} \mathcal{M}_{2}^{\mu},
$$

where $\mathcal{J}_{\perp}$ denotes the exchange interaction between spin in the QSL $S_{i, l}^{\mu}$ and the magnetic moment of the ferromagnets $\mathcal{M}_{l}^{\mu}$, with $l=1,2$ labeling the two different magnets and QSL layers. We consider sufficiently small $\mathcal{J}_{\perp}$ that does not lead to many-body reconstruction. In such case, the mean-field solution of Eq. (2) remains, and the effect of the ferromagnets can be projected onto the spinon mean-field Hamiltonian as

$$
H^{\prime}=H+\frac{1}{2} \sum_{i, \mu, l, s, s^{\prime}} \mathcal{J}_{\perp} \sigma_{s, s^{\prime}}^{\mu} \mathcal{M}_{l}^{\mu} f_{i, s, l}^{\dagger} f_{i, s^{\prime}, l} .
$$

In the case of the antiferromagnetic alignment as depicted in Fig. 3(a), the magnetic encapsulation creates an effective spin-dependent interlayer spinon bias $J=\mathcal{J}_{\perp}\left(\mathcal{M}_{1}^{z}-\mathcal{M}_{2}^{z}\right)$ on the twisted Dirac QSL [51,72,90]. The effective interlayer 
(a)

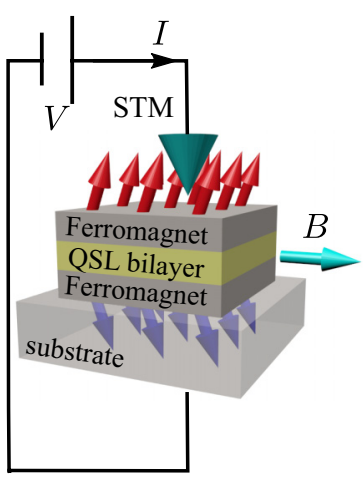

(c)

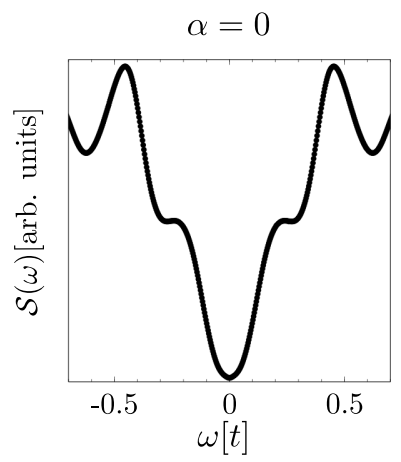

(b)

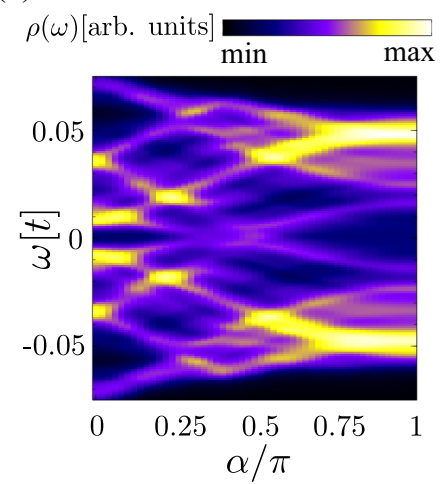

(d)

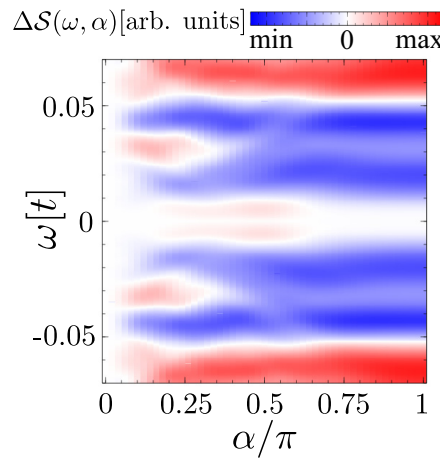

FIG. 4. (a) Sketch of the experimental setup for probing QSL via inelastic spectroscopy. (b) Spinon DOS of the twisted QSL at $\theta=$ $0.93^{\circ}$ and $J=0.1 t$ under different $\alpha(B)$. (c) Spin structure factor $\mathcal{S}(\omega)$ with $\alpha=0$. (d) Change in spin structure factor $\Delta \mathcal{S}(\omega, \alpha)$ for different $\alpha$.

spinon bias $J$ induced by proximity exchange fields has a dramatic impact on the low energy spinon band structure of the twisted Dirac QSL. Due to the broken mirror symmetry of the twisted QSL [94], the exchange bias causes spin-splitting in the spinon band structure in reciprocal space [Fig. 3(b)]. At the flat band twisting angle, the gap at Fermi level drastically increases with $J$ [Fig. 3(c)]. When the twisting angle is not the flat one, the spinon DOS gets substantially modified at larger exchange couplings [Fig. 3(d)].

We now move on to discuss how the impact of the exchange bias can be used for the detection of the spinon spectra. Scanning tunnel spectroscopy can be exploited to detect spin excitations by means of inelastic transport $[9,10,41,95]$. We now consider a similar setup, in which the magnetically encapsulated QSL bilayer is explored by means of vertical transport with STM as shown in Fig. 4(a). From the experimental point of view, spinon states will emerge in the inelastic contribution to the current, and will appear as peaks in $d^{2} I / d V^{2}$ when phonon contributions are neglected [96]. In such case, the measured $d^{2} I / d V^{2}$ is proportional to the spin structure factor $\mathcal{S}(\omega)$, the latter being a convolution of spinon DOS [95,97]: $\mathcal{S}(\omega)=\int d \omega_{1} d \omega_{2} \frac{\rho\left(\omega_{1}\right) \rho\left(\omega_{2}\right)}{\omega+\omega_{1}-\omega_{2}+i 0^{+}}\left[f\left(\omega_{1}\right)-\right.$ $f\left(\omega_{2}\right)$ ] where $f(\omega)$ is the Fermi-Dirac distribution.

To reveal the impact of the magnetic encapsulation, we now consider the change of the signal with respect to an in-plane magnetic field $B$, that is used to control the direction of magnetism in the magnets. The magnetic field will tune the

angle between magnetization of the two magnets from $\pi$ to $\pi-\alpha(B)$, and modifies spinon DOS in the QSL bilayer due to proximity effect. For twisting angle $\theta=0.93^{\circ}$, and effective exchange bias $J=0.1 t$, the spinon DOS under different $\alpha(B)$ is shown in Fig. 4(b). The modified spinon DOS exhibits peaks at different frequencies than the original one. Taking the specific case of antiferromagnetic alignment $\alpha=0$, it is seen that the spin structure factor has peak structures, inherited from the peaks in the spinon DOS [Fig. 4(c)]. Due to the modification of the spinon DOS with the field, an analogous effect is expected in the $d^{2} I / d V^{2}$. For this sake, we now compute the change in the $d^{2} I / d V^{2}$ as a function of the magnetic field, defined as $\Delta \mathcal{S}(\omega, \alpha)=\mathcal{S}(\omega, \alpha)-\mathcal{S}(\omega, 0)$, and shown in Fig. 4(d). In particular, we see in Fig. 4(d) the existence of deeps and peaks in the differential $d^{2} I / d V^{2}$, manifesting from the dramatic change of spinon DOS with the magnetic field in Fig. 4(b).

\section{DISCUSSION}

Finally, we comment on specific quantitative aspects of our proposal relevant for experiments. First, in our paper, we have considered exchange couplings between the two monolayer QSLs on the order of $J_{\perp} \approx 0.3 J_{\|}$. Due to the scaling relation as proved for our system in Appendix A, our results apply to systems with smaller exchange couplings $J_{\perp}$ at smaller twisting angles. The specific prediction of such exchange coupling should be performed via first principle methods for the specific materials considered [98,99], and could ultimately be controlled with pressure [100,101]. For our phenomenology, a change in the exchange coupling just drifts the physics towards bigger or smaller angles, yet without qualitatively changing the overall behavior [102]. Second, when exchange proximity is considered, the exchange proximity must be smaller than the intralayer exchange to not perturb the QSL ground state. We note that for smaller values of exchange proximity than what we considered here, the reconstruction of spinon DOS remains, as shown in Fig. 6. Third, in order to tilt the direction of the magnets by an external magnetic field, yet without breaking the QSL ground state, a soft magnetic axis is preferred. As a reference, taking $\mathrm{CrBr}_{3}$ as the ferromagnet, the anisotropy energy can be overcome with a magnetic field of around $1 \mathrm{~T}$ [103], whose Zeeman energy scale of $0.04 \mathrm{meV}$ is not expected to perturb QSLs with exchange constants on the order of $5 \mathrm{meV}[19,104]$, and in particular for $\mathrm{TaS}_{2}$ on the order of $\sim 100 \mathrm{meV}$ [17]. The application of a magnetic field is also expected to affect the magnons of the ferromagnetic encapsulation, yet those states will contribute with a uniform background to the $d^{2} I / d V^{2}$ signal that can be subtracted $[9,10]$. Finally, although our analysis has focused on a Dirac QSL, analogous calculations can be performed with other QSL ground states, such as the gaped QSL of $\mathrm{RuCl}_{3}$ [25].

We also note that our results focus on a spinon meanfield treatment to capture the nature of the spinon excitations in the twisted heterostructure. While an exact treatment of our system with pure many-body methods would be greatly interesting, this is well beyond the capability of the stateof-the-art techniques for frustrated two-dimensional quantum many-body models, such as density matrix renormalization group (DMRG) [65,105-107]. 
(a)

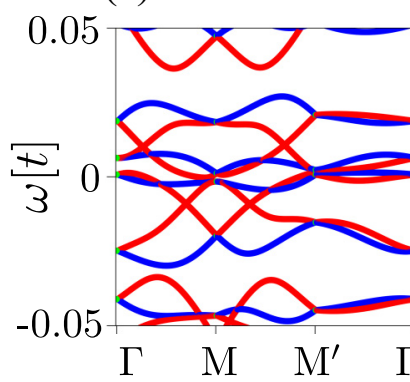

(c)

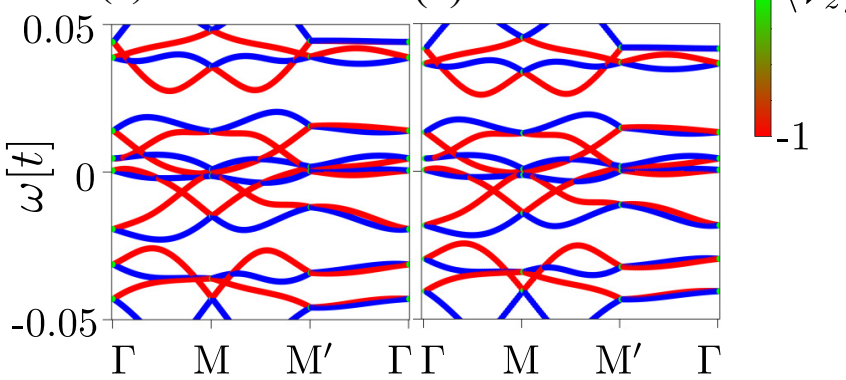

FIG. 5. Spinon band structure of the twisted Dirac QSL at different twisting angles $\theta=1.30^{\circ}, 1.12^{\circ}, 0.99^{\circ}, 0.93^{\circ}$ [from (a) to (d)] with the same effective angle $\theta_{\text {eff }}$.

\section{CONCLUSION}

To summarize, we have shown that a van der Waals heterostructure based on a magnetically encapsulated bilayer QSL allows designing controllable spinon physics, and ultimately detecting moire spinons. We showed that a twisted bilayer QSL gives rise to a topological gap opening purely driven by the twist, and in fine-tuned regimes spinon flat bands. Furthermore, we showed that encapsulating the twisted QSL bilayer with ferromagnets produces exchange bias via proximity effect, which allows us to magnetically control the spinon band structure. Based on such magnetic tunability, we proposed an experimental identification of QSL phases, utilizing small magnetic field together with inelastic spectroscopy. Our results put forward twist engineering as a means (a)

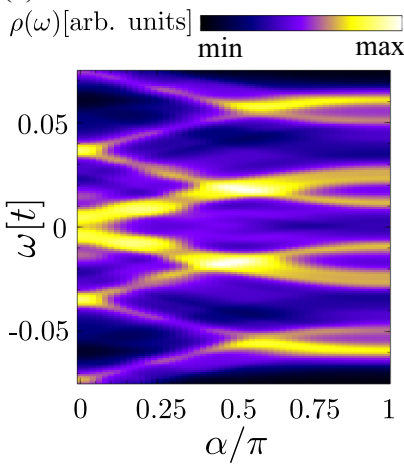

(b)

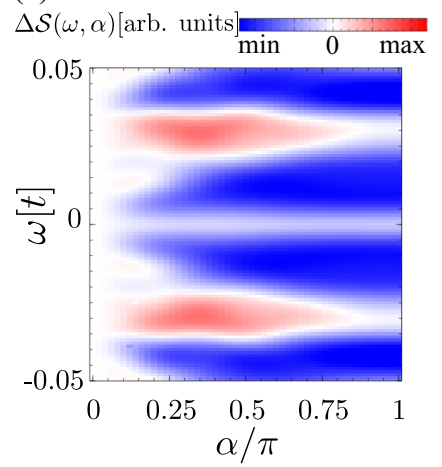

FIG. 6. (a) Spinon DOS of the twisted QSL at $\theta=0.93^{\circ}$ and $J=0.05 t$ under different $\alpha(B)$. (b) Change in spin structure factor $\Delta \mathcal{S}(\omega, \alpha)$ for different $\alpha$. of exploiting exotic spinon phenomena in quantum spin liquids, highlighting the versatility of magnetic van der Waals heterostructures to explore emergent spinon phenomena.

\section{ACKNOWLEDGMENTS}

We acknowledge the computational resources provided by the Aalto Science-IT project, and the financial support from the Academy of Finland Projects No. 331342 and No. 336243. We thank P. Liljeroth, S. Kezilebieke, V. Vaňo, and S. Ganguli for fruitful discussions.

\section{APPENDIX A: SCALING RELATION IN TWISTED QUANTUM SPIN LIQUID}

When computing the band structure of the twisted quantum spin liquid (QSL) in the main text, we used a scaling relation for computational convenience. We justify the usage of such a scaling relation in this section.

The scaling relation states that the band structure of a Dirac system is related to the magnitude of the hybridization between two Dirac cones that have a certain momentum difference, and that are coupled by a certain energy scale. When used in twisted bilayer systems, the scaling relation states that the band structure of the twisted bilayer depends only on the effective angle $\theta_{\text {eff }}=\theta \times \frac{t_{0}}{t_{\perp}}$, where $\theta$ is the twisting angle, $t_{\perp}$ is the interlayer coupling strength, and $t_{0}$ is a constant. The validity of this scaling relation in a lattice model relies on taking interlayer couplings that are small in comparison with the bandwidth of the associated Dirac cone. This condition simply stems from the fact that, in the Dirac approximation, the hybridization between the Dirac cones solely depends on the relative scales between hybridization strength and momentum mismatch $[69,102]$. This same argument, originally developed for twisted bilayer graphene [69,102], can be also extended to generic Dirac systems. For the sake of concreteness, we now explicitly show how the scaling relation also holds for our twisted quantum spin liquid, which is a $\pi$-flux Dirac system. We show in Fig. 5 the spinon band structure of the twisted quantum spin-liquid at four different $\theta$ and $t_{\perp}$, keeping the ratio $\theta_{\text {eff }}$ constant. The band structure is the same for all $\theta$ except for a renormalization of the energy scale, that is given by the used interlayer coupling. This is exactly the same phenomenology as in the rescaling of twisted graphene bilayers, demonstrating that the scaling relation also holds for the twisted Dirac quantum spin liquid in the main text.

\section{APPENDIX B: RECONSTRUCTION OF SPINON SPECTRA WITH SMALLER EXCHANGE PROXIMITY}

In the main text, we showed the reconstruction of the spinon spectra in the twisted QSL in proximity to ferromagnetic insulators with effective coupling $J=0.1 t$ and under an in-plane magnetic field. We noted that the exchange proximity should not perturb the QSL ground state. For the $U(1)$ Dirac QSL, which survives in a large phase space in a Heisenberg model on the triangular lattice $[65,108], J=0.1 t$ is not likely to break a QSL ground state deep in the phase space. Yet, for QSL states lying at the boundary of the phase space and QSL 
states that survive in a small phase space such as the Kitaev spin liquid, smaller $J$ is preferred to preserve the QSL state. For such sake, we show that the reconstruction of the spinon spectra and the corresponding changes in the spin structure factor persists at $J=0.05 t$ (Fig. 6), allowing our proposal to be applied to more generic cases.
[1] J.-G. Park, Opportunities and challenges of $2 \mathrm{~d}$ magnetic van der waals materials: magnetic graphene? J. Phys.: Condens. Matter 28, 301001 (2016).

[2] B. Huang, G. Clark, E. Navarro-Moratalla, D. R. Klein, R. Cheng, K. L. Seyler, D. Zhong, E. Schmidgall, M. A. McGuire, D. H. Cobden, W. Yao, D. Xiao, P. Jarillo-Herrero, and $\mathrm{X} . \mathrm{Xu}$, Layer-dependent ferromagnetism in a van der waals crystal down to the monolayer limit, Nature (London) 546, 270 (2017).

[3] C. Gong, L. Li, Z. Li, H. Ji, A. Stern, Y. Xia, T. Cao, W. Bao, C. Wang, Y. Wang, Z. Q. Qiu, R. J. Cava, S. G. Louie, J. Xia, and X. Zhang, Discovery of intrinsic ferromagnetism in twodimensional van der waals crystals, Nature (London) 546, 265 (2017).

[4] M. Gibertini, M. Koperski, A. F. Morpurgo, and K. S. Novoselov, Magnetic 2d materials and heterostructures, Nat. Nanotechnol. 14, 408 (2019).

[5] Y. Deng, Y. Yu, Y. Song, J. Zhang, N. Z. Wang, Z. Sun, Y. Yi, Y. Z. Wu, S. Wu, J. Zhu, J. Wang, X. H. Chen, and Y. Zhang, Gate-tunable room-temperature ferromagnetism in two-dimensional fe3gete2, Nature (London) 563, 94 (2018).

[6] B. Huang, G. Clark, D. R. Klein, D. MacNeill, E. NavarroMoratalla, K. L. Seyler, N. Wilson, M. A. McGuire, D. H. Cobden, D. Xiao, W. Yao, P. Jarillo-Herrero, and X. Xu, Electrical control of $2 \mathrm{~d}$ magnetism in bilayer CrI3, Nat. Nanotechnol. 13, 544 (2018).

[7] S. Jiang, L. Li, Z. Wang, K. F. Mak, and J. Shan, Controlling magnetism in $2 \mathrm{~d} \mathrm{CrI3}$ by electrostatic doping, Nat. Nanotechnol. 13, 549 (2018).

[8] N. Sivadas, S. Okamoto, and D. Xiao, Gate-Controllable Magneto-Optic Kerr Effect in Layered Collinear Antiferromagnets, Phys. Rev. Lett. 117, 267203 (2016).

[9] D. Ghazaryan, M. T. Greenaway, Z. Wang, V. H. GuarochicoMoreira, I. J. Vera-Marun, J. Yin, Y. Liao, S. V. Morozov, O. Kristanovski, A. I. Lichtenstein, M. I. Katsnelson, F. Withers, A. Mishchenko, L. Eaves, A. K. Geim, K. S. Novoselov, and A. Misra, Magnon-assisted tunnelling in van der waals heterostructures based on $\mathrm{CrBr} 3$, Nat. Electron. 1, 344 (2018).

[10] D. R. Klein, D. MacNeill, J. L. Lado, D. Soriano, E. NavarroMoratalla, K. Watanabe, T. Taniguchi, S. Manni, P. Canfield, J. Fernández-Rossier, and P. Jarillo-Herrero, Probing magnetism in $2 \mathrm{~d}$ van der waals crystalline insulators via electron tunneling, Science 360, 1218 (2018).

[11] T. Song, X. Cai, M. W.-Y. Tu, X. Zhang, B. Huang, N. P. Wilson, K. L. Seyler, L. Zhu, T. Taniguchi, K. Watanabe, M. A. McGuire, D. H. Cobden, D. Xiao, W. Yao, and X. Xu, Giant tunneling magnetoresistance in spin-filter van der waals heterostructures, Science 360, 1214 (2018).

[12] S. Jiang, L. Li, Z. Wang, J. Shan, and K. F. Mak, Spin transistor built on $2 \mathrm{~d}$ van der waals heterostructures, Nat. Electron. 2, 159 (2019).

[13] S. Kezilebieke, M. N. Huda, V. Vaňo, M. Aapro, S. C. Ganguli, O. J. Silveira, S. Głodzik, A. S. Foster, T. Ojanen, and P.
Liljeroth, Topological superconductivity in a van der waals heterostructure, Nature (London) 588, 424 (2020).

[14] S. Kezilebieke, V. Vaňo, M. N. Huda, M. Aapro, S. C. Ganguli, P. Liljeroth, and J. L. Lado, Moiré-enabled topological superconductivity, arXiv:2011.09760.

[15] D. Zhong, K. L. Seyler, X. Linpeng, R. Cheng, N. Sivadas, B. Huang, E. Schmidgall, T. Taniguchi, K. Watanabe, M. A. McGuire, W. Yao, D. Xiao, K.-M. C. Fu, and X. Xu, Van der waals engineering of ferromagnetic semiconductor heterostructures for spin and valleytronics, Sci. Adv. 3, e1603113 (2017).

[16] K. T. Law and P. A. Lee, 1t-TaS2 as a quantum spin liquid, Proc. Natl. Acad. Sci. U.S.A. 114, 6996 (2017).

[17] M. Klanjšek, A. Zorko, R. Žitko, J. Mravlje, Z. Jagličić, P. K. Biswas, P. Prelovšek, D. Mihailovic, and D. Arčon, A high-temperature quantum spin liquid with polaron spins, Nat. Phys. 13, 1130 (2017).

[18] Y. Chen, W. Ruan, M. Wu, S. Tang, H. Ryu, H.-Z. Tsai, R. Lee, S. Kahn, F. Liou, C. Jia, O. R. Albertini, H. Xiong, T. Jia, Z. Liu, J. A. Sobota, A. Y. Liu, J. E. Moore, Z.-X. Shen, S. G. Louie, S.-K. Mo et al., Strong correlations and orbital texture in single-layer 1t-TaSe2, Nat. Phys. 16, 218 (2020).

[19] A. Banerjee, C. A. Bridges, J.-Q. Yan, A. A. Aczel, L. Li, M. B. Stone, G. E. Granroth, M. D. Lumsden, Y. Yiu, J. Knolle, S. Bhattacharjee, D. L. Kovrizhin, R. Moessner, D. A. Tennant, D. G. Mandrus, and S. E. Nagler, Proximate kitaev quantum spin liquid behaviour in a honeycomb magnet, Nat. Mater. 15, 733 (2016).

[20] L. Balents, Spin liquids in frustrated magnets, Nature (London) 464, 199 (2010).

[21] P. A. Lee, An end to the drought of quantum spin liquids, Science 321, 1306 (2008).

[22] C. Broholm, R. J. Cava, S. A. Kivelson, D. G. Nocera, M. R. Norman, and T. Senthil, Quantum spin liquids, Science 367, eaay0668 (2020).

[23] Y. Zhou, K. Kanoda, and T.-K. Ng, Quantum spin liquid states, Rev. Mod. Phys. 89, 025003 (2017).

[24] L. Savary and L. Balents, Quantum spin liquids: a review, Rep. Prog. Phys. 80, 016502 (2016).

[25] A. Kitaev, Anyons in an exactly solved model and beyond, Ann. Phys. 321, 2 (2006).

[26] P. W. Anderson, The resonating valence bond state in la2cuo4 and superconductivity, Science 235, 1196 (1987).

[27] Z. A. Kelly, M. J. Gallagher, and T. M. McQueen, Electron Doping a Kagome Spin Liquid, Phys. Rev. X 6, 041007 (2016).

[28] T.-H. Han, J. S. Helton, S. Chu, D. G. Nocera, J. A. RodriguezRivera, C. Broholm, and Y. S. Lee, Fractionalized excitations in the spin-liquid state of a kagome-lattice antiferromagnet, Nature (London) 492, 406 (2012).

[29] M. Fu, T. Imai, T.-H. Han, and Y. S. Lee, Evidence for a gapped spin-liquid ground state in a kagome heisenberg antiferromagnet, Science 350, 655 (2015). 
[30] B. J. Powell and R. H. McKenzie, Quantum frustration in organic mott insulators: From spin liquids to unconventional superconductors, Rep. Prog. Phys. 74, 056501 (2011).

[31] S. K. Takahashi, J. Wang, A. Arsenault, T. Imai, M. Abramchuk, F. Tafti, and P. M. Singer, Spin Excitations of a Proximate Kitaev Quantum Spin Liquid Realized in $\mathrm{cu}_{2} \mathrm{iro}_{3}$, Phys. Rev. X 9, 031047 (2019).

[32] M. R. Norman, Colloquium: Herbertsmithite and the search for the quantum spin liquid, Rev. Mod. Phys. 88, 041002 (2016).

[33] H. Takagi, T. Takayama, G. Jackeli, G. Khaliullin, and S. E. Nagler, Concept and realization of kitaev quantum spin liquids, Nat. Rev. Phys. 1, 264 (2019).

[34] Y. Shimizu, K. Miyagawa, K. Kanoda, M. Maesato, and G. Saito, Spin Liquid State in An Organic Mott Insulator with a Triangular Lattice, Phys. Rev. Lett. 91, 107001 (2003).

[35] M. Yamashita, N. Nakata, Y. Kasahara, T. Sasaki, N. Yoneyama, N. Kobayashi, S. Fujimoto, T. Shibauchi, and Y. Matsuda, Thermal-transport measurements in a quantum spin-liquid state of the frustrated triangular magnet $\kappa$-(BEDTTTF)2cu2(CN)3, Nat. Phys. 5, 44 (2008).

[36] T. Itou, A. Oyamada, S. Maegawa, M. Tamura, and R. Kato, Quantum spin liquid in the spin-1/2 triangular antiferromagnet $\mathrm{Etme}_{3} \mathrm{Sb}\left[\mathrm{Pd}(\mathrm{dmit})_{2}\right]_{2}$, Phys. Rev. B 77, 104413 (2008).

[37] T. Isono, H. Kamo, A. Ueda, K. Takahashi, M. Kimata, H. Tajima, S. Tsuchiya, T. Terashima, S. Uji, and H. Mori, Gapless Quantum Spin Liquid in An Organic Spin-1/2 Triangular-Lattice $\kappa-\mathrm{h}_{3}$ (cat-edt-ttf) ${ }_{2}$, Phys. Rev. Lett. 112, 177201 (2014)

[38] J. S. Helton, K. Matan, M. P. Shores, E. A. Nytko, B. M. Bartlett, Y. Yoshida, Y. Takano, A. Suslov, Y. Qiu, J.-H. Chung, D. G. Nocera, and Y. S. Lee, Spin Dynamics of the Spin-1/2 Kagome Lattice Antiferromagnet $\mathrm{zncu}_{3}(\mathrm{OH})_{6} \mathrm{Cl}_{2}$, Phys. Rev. Lett. 98, 107204 (2007).

[39] L. Ding, P. Manuel, S. Bachus, F. Grußler, P. Gegenwart, J. Singleton, R. D. Johnson, H. C. Walker, D. T. Adroja, A. D. Hillier, and A. A. Tsirlin, Gapless spin-liquid state in the structurally disorder-free triangular antiferromagnet naybo ${ }_{2}$, Phys. Rev. B 100, 144432 (2019).

[40] M. M. Bordelon, E. Kenney, C. Liu, T. Hogan, L. Posthuma, M. Kavand, Y. Lyu, M. Sherwin, N. P. Butch, C. Brown, M. J. Graf, L. Balents, and S. D. Wilson, Field-tunable quantum disordered ground state in the triangular-lattice antiferromagnet NaYbO2, Nat. Phys. 15, 1058 (2019).

[41] E. J. König, M. T. Randeria, and B. Jäck, Tunneling Spectroscopy of Quantum Spin Liquids, Phys. Rev. Lett. 125, 267206 (2020)

[42] J. Feldmeier, W. Natori, M. Knap, and J. Knolle, Local probes for charge-neutral edge states in two-dimensional quantum magnets, Phys. Rev. B 102, 134423 (2020).

[43] E. Suárez Morell, J. D. Correa, P. Vargas, M. Pacheco, and Z. Barticevic, Flat bands in slightly twisted bilayer graphene: Tight-binding calculations, Phys. Rev. B 82, 121407(R) (2010).

[44] R. Bistritzer and A. H. MacDonald, Moire bands in twisted double-layer graphene, Proc. Natl. Acad. Sci. U.S.A. 108, 12233 (2011).

[45] Y. Cao, V. Fatemi, A. Demir, S. Fang, S. L. Tomarken, J. Y. Luo, J. D. Sanchez-Yamagishi, K. Watanabe, T. Taniguchi, E. Kaxiras, R. C. Ashoori, and P. Jarillo-Herrero, Correlated insulator behaviour at half-filling in magic-angle graphene superlattices, Nature (London) 556, 80 (2018).

[46] Y. Cao, V. Fatemi, S. Fang, K. Watanabe, T. Taniguchi, E. Kaxiras, and P. Jarillo-Herrero, Unconventional superconductivity in magic-angle graphene superlattices, Nature (London) 556, 43 (2018).

[47] X. Lu, P. Stepanov, W. Yang, M. Xie, M. A. Aamir, I. Das, C. Urgell, K. Watanabe, T. Taniguchi, G. Zhang, A. Bachtold, A. H. MacDonald, and D. K. Efetov, Superconductors, orbital magnets and correlated states in magic-angle bilayer graphene, Nature (London) 574, 653 (2019).

[48] M. Yankowitz, S. Chen, H. Polshyn, Y. Zhang, K. Watanabe, T. Taniguchi, D. Graf, A. F. Young, and C. R. Dean, Tuning superconductivity in twisted bilayer graphene, Science $\mathbf{3 6 3}$, 1059 (2019).

[49] Y. Cao, D. Chowdhury, D. Rodan-Legrain, O. Rubies-Bigorda, K. Watanabe, T. Taniguchi, T. Senthil, and P. Jarillo-Herrero, Strange Metal in Magic-Angle Graphene with Near Planckian Dissipation, Phys. Rev. Lett. 124, 076801 (2020).

[50] Y. Cao, D. Rodan-Legrain, O. Rubies-Bigorda, J. M. Park, K. Watanabe, T. Taniguchi, and P. Jarillo-Herrero, Tunable correlated states and spin-polarized phases in twisted bilayerbilayer graphene, Nature (London) 583, 215 (2020).

[51] T. M. R. Wolf, O. Zilberberg, G. Blatter, and J. L. Lado, Spontaneous Valley Spirals in Magnetically Encapsulated Twisted Bilayer Graphene, Phys. Rev. Lett. 126, 056803 (2021).

[52] T. M. R. Wolf, J. L. Lado, G. Blatter, and O. Zilberberg, Electrically Tunable Flat Bands and Magnetism in Twisted Bilayer Graphene, Phys. Rev. Lett. 123, 096802 (2019).

[53] M. Koshino, Band structure and topological properties of twisted double bilayer graphene, Phys. Rev. B 99, 235406 (2019).

[54] Z. Liu, A. Abouelkomsan, and E. J. Bergholtz, Gate-Tunable Fractional Chern Insulators in Twisted Double Bilayer Graphene, Phys. Rev. Lett. 126, 026801 (2021).

[55] A. Abouelkomsan, Z. Liu, and E. J. Bergholtz, Particle-Hole Duality, Emergent Fermi Liquids, and Fractional Chern Insulators in Moiré Flatbands, Phys. Rev. Lett. 124, 106803 (2020).

[56] M. Serlin, C. L. Tschirhart, H. Polshyn, Y. Zhang, J. Zhu, K. Watanabe, T. Taniguchi, L. Balents, and A. F. Young, Intrinsic quantized anomalous hall effect in a moiré heterostructure, Science 367, 900 (2019).

[57] Y.-H. Zhang and T. Senthil, Quantum hall spin liquids and their possible realization in moiré systems, Phys. Rev. B 102, 115127 (2020).

[58] O. Can, T. Tummuru, R. P. Day, I. Elfimov, A. Damascelli, and M. Franz, High-temperature topological superconductivity in twisted double-layer copper oxides, Nat. Phys. 17, 519 (2021).

[59] K. Hejazi, Z.-X. Luo, and L. Balents, Noncollinear phases in moiré magnets, Proc. Natl. Acad. Sci. U.S.A. 117, 10721 (2020).

[60] J. May-Mann and T. L. Hughes, Twisted kitaev bilayers and the moiré ising model, Phys. Rev. B 101, 245126 (2020).

[61] X. Zhu, H. Guo, and S. Feng, Magnon bands in twisted bilayer honeycomb quantum magnets, Chinese Phys. B 30, 077505 (2021).

[62] H. Murayama, Y. Sato, T. Taniguchi, R. Kurihara, X. Z. Xing, W. Huang, S. Kasahara, Y. Kasahara, I. Kimchi, M. Yoshida, Y. Iwasa, Y. Mizukami, T. Shibauchi, M. Konczykowski, and 
Y. Matsuda, Effect of quenched disorder on the quantum spin liquid state of the triangular-lattice antiferromagnet $1 t-\operatorname{tas}_{2}$, Phys. Rev. Research 2, 013099 (2020).

[63] S. Mañas-Valero, B. Huddart, T. Lancaster, E. Coronado, and F. Pratt, Multiple quantum spin liquid phases in 1T-TaS2, npj Quantum Mater. 6, 69 (2021).

[64] Y. Iqbal, W.-J. Hu, R. Thomale, D. Poilblanc, and F. Becca, Spin liquid nature in the heisenberg $J_{1}-J_{2}$ triangular antiferromagnet, Phys. Rev. B 93, 144411 (2016).

[65] S. Hu, W. Zhu, S. Eggert, and Y.-C. He, Dirac Spin Liquid on the Spin-1/2 Triangular Heisenberg Antiferromagnet, Phys. Rev. Lett. 123, 207203 (2019).

[66] Here we choose a unit-cell containing $2 \times 2$ sites.

[67] A. O. Sboychakov, A. L. Rakhmanov, A. V. Rozhkov, and F. Nori, Electronic spectrum of twisted bilayer graphene, Phys. Rev. B 92, 075402 (2015).

[68] M.-H. Liu, P. Rickhaus, P. Makk, E. Tóvári, R. Maurand, F. Tkatschenko, M. Weiss, C. Schönenberger, and K. Richter, Scalable Tight-Binding Model for Graphene, Phys. Rev. Lett. 114, 036601 (2015).

[69] L. A. Gonzalez-Arraga, J. L. Lado, F. Guinea, and P. SanJose, Electrically Controllable Magnetism in Twisted Bilayer Graphene, Phys. Rev. Lett. 119, 107201 (2017).

[70] Details of the scaling relation are given in Appendix A.

[71] E. Colomés and M. Franz, Antichiral Edge States in A Modified Haldane Nanoribbon, Phys. Rev. Lett. 120, 086603 (2018).

[72] D. Soriano and J. L. Lado, Exchange-bias controlled correlations in magnetically encapsulated twisted van der waals dichalcogenides, J. Phys. D 53, 474001 (2020).

[73] A. Ramires and J. L. Lado, Impurity-induced triple point fermions in twisted bilayer graphene, Phys. Rev. B 99, 245118 (2019).

[74] A. Lopez-Bezanilla and J. L. Lado, Electrical band flattening, valley flux, and superconductivity in twisted trilayer graphene, Phys. Rev. Research 2, 033357 (2020).

[75] Y. Ran, M. Hermele, P. A. Lee, and X.-G. Wen, ProjectedWave-Function Study of the Spin-1/2 Heisenberg Model on the Kagomé Lattice, Phys. Rev. Lett. 98, 117205 (2007).

[76] J. Ahn, S. Park, and B.-J. Yang, Failure of Nielsen-Ninomiya Theorem and Fragile Topology in Two-Dimensional Systems with Space-Time Inversion Symmetry: Application to Twisted Bilayer Graphene at Magic Angle, Phys. Rev. X 9, 021013 (2019).

[77] H. C. Po, L. Zou, A. Vishwanath, and T. Senthil, Origin of Mott Insulating Behavior and Superconductivity in Twisted Bilayer Graphene, Phys. Rev. X 8, 031089 (2018).

[78] QuanSheng Wu, J. Liu, Y. Guan, and O. V. Yazyev, Landau Levels as a Probe for Band Topology in Graphene Moiré Superlattices, Phys. Rev. Lett. 126, 056401 (2021).

[79] J. Liu, Z. Ma, J. Gao, and X. Dai, Quantum Valley Hall Effect, Orbital Magnetism, and Anomalous Hall Effect in Twisted Multilayer Graphene Systems, Phys. Rev. X 9, 031021 (2019).

[80] N. R. Chebrolu, B. L. Chittari, and J. Jung, Flat bands in twisted double bilayer graphene, Phys. Rev. B 99, 235417 (2019).

[81] E. Prada, P. San-Jose, L. Brey, and H. A. Fertig, Band topology and the quantum spin hall effect in bilayer graphene, Solid State Commun. 151, 1075 (2011).
[82] F. Zhang, A. H. MacDonald, and E. J. Mele, Valley chern numbers and boundary modes in gapped bilayer graphene, Proc. Natl. Acad. Sci. U.S.A. 110, 10546 (2013).

[83] A. R. Wright and T. Hyart, Robust one-dimensional wires in lattice mismatched bilayer graphene, Appl. Phys. Lett. 98, 251902 (2011).

[84] P. San-Jose and E. Prada, Helical networks in twisted bilayer graphene under interlayer bias, Phys. Rev. B 88, 121408(R) (2013).

[85] P. Rickhaus, J. Wallbank, S. Slizovskiy, R. Pisoni, H Overweg, Y. Lee, M. Eich, M.-H. Liu, K. Watanabe, T. Taniguchi, T. Ihn, and K. Ensslin, Transport through a network of topological channels in twisted bilayer graphene, Nano Lett. 18, 6725 (2018).

[86] A. F. Morpurgo and F. Guinea, Intervalley Scattering, LongRange Disorder, and Effective Time-Reversal Symmetry Breaking in Graphene, Phys. Rev. Lett. 97, 196804 (2006).

[87] J.-H. Chen, W. G. Cullen, C. Jang, M. S. Fuhrer, and E. D. Williams, Defect Scattering in Graphene, Phys. Rev. Lett. 102, 236805 (2009).

[88] S. Kezilebieke, O. J. Silveira, M. N. Huda, V. Vaňo, M. Aapro, S. C. Ganguli, J. Lahtinen, R. Mansell, S. van Dijken, A. S. Foster, and P. Liljeroth, Electronic and magnetic characterization of epitaxial crbr $_{3}$ monolayers, Adv. Mater. 33, 2006850 (2021).

[89] P. W. Anderson, Antiferromagnetism. theory of superexchange interaction, Phys. Rev. 79, 350 (1950).

[90] C. Cardoso, D. Soriano, N. A. García-Martínez, and J. Fernández-Rossier, Van der Waals Spin Valves, Phys. Rev. Lett. 121, 067701 (2018).

[91] S. Singh, J. Katoch, T. Zhu, K.-Y. Meng, T. Liu, J. T. Brangham, F. Yang, M. E. Flatté, and R. K. Kawakami, Strong Modulation of Spin Currents in Bilayer Graphene by Static and Fluctuating Proximity Exchange Fields, Phys. Rev. Lett. 118, 187201 (2017).

[92] P. Wei, S. Lee, F. Lemaitre, L. Pinel, D. Cutaia, W. Cha, F. Katmis, Y. Zhu, D. Heiman, J. Hone, J. S. Moodera, and C.-T. Chen, Strong interfacial exchange field in the graphene/EuS heterostructure, Nat. Mater. 15, 711 (2016).

[93] Z. Wang, C. Tang, R. Sachs, Y. Barlas, and J. Shi, ProximityInduced Ferromagnetism in Graphene Revealed by the Anomalous Hall Effect, Phys. Rev. Lett. 114, 016603 (2015).

[94] The mirror symmetry $M_{x}\left(M_{y}\right)$ is present if the system is invariant when mirrored by the $x(y)$ plane and then exchanging the top and bottom layers.

[95] G. Chen and J. L. Lado, Impurity-induced resonant spinon zero modes in dirac quantum spin liquids, Phys. Rev. Research 2, 033466 (2020)

[96] Spinon energy scale is smaller than the magnon gap in ferromagnets, allowing us to neglect the contribution from inelastic scattering with magnons.

[97] A. Spinelli, B. Bryant, F. Delgado, J. Fernández-Rossier, and A. F. Otte, Imaging of spin waves in atomically designed nanomagnets, Nat. Mater. 13, 782 (2014).

[98] N. Sivadas, S. Okamoto, X. Xu, C. J. Fennie, and D. Xiao, Stacking-dependent magnetism in bilayer CrI3, Nano Lett. 18, 7658 (2018).

[99] D. Soriano, C. Cardoso, and J. Fernández-Rossier, Interplay between interlayer exchange and stacking in CrI3 bilayers, Solid State Commun. 299, 113662 (2019). 
[100] T. Song, Z. Fei, M. Yankowitz, Z. Lin, Q. Jiang, K. Hwangbo, Q. Zhang, B. Sun, T. Taniguchi, K. Watanabe, M. A. McGuire, D. Graf, T. Cao, J.-H. Chu, D. H. Cobden, C. R. Dean, D. Xiao, and $\mathrm{X}$. Xu, Switching $2 \mathrm{~d}$ magnetic states via pressure tuning of layer stacking, Nat. Mater. 18, 1298 (2019).

[101] T. Li, S. Jiang, N. Sivadas, Z. Wang, Y. Xu, D. Weber, J. E. Goldberger, K. Watanabe, T. Taniguchi, C. J. Fennie, K. F. Mak, and J. Shan, Pressure-controlled interlayer magnetism in atomically thin CrI3, Nat. Mater. 18, 1303 (2019).

[102] J. M. B. Lopes dos Santos, N. M. R. Peres, and A. H. Castro Neto, Graphene Bilayer with A Twist: Electronic Structure, Phys. Rev. Lett. 99, 256802 (2007).

[103] N. Richter, D. Weber, F. Martin, N. Singh, U. Schwingenschlögl, B. V. Lotsch, and M. Kläui, Temperature-dependent magnetic anisotropy in the layered magnetic semiconductors $\mathrm{Cri}_{3}$ and $\mathrm{CrBr}_{3}$, Phys. Rev. Materials 2, 024004 (2018).
[104] L. J. Sandilands, Y. Tian, K. W. Plumb, Y.-J. Kim, and K. S. Burch, Scattering Continuum and Possible Fractionalized Excitations in $\alpha-\mathrm{rucl}_{3}$, Phys. Rev. Lett. 114, 147201 (2015).

[105] U. Schollwöck, The density-matrix renormalization group, Rev. Mod. Phys. 77, 259 (2005).

[106] E. M. Stoudenmire and S. R. White, Studying twodimensional systems with the density matrix renormalization group, Annu. Rev. Condens. Matter Phys. 3, 111 (2012).

[107] A. Szasz, J. Motruk, M. P. Zaletel, and J. E. Moore, Chiral Spin Liquid Phase of the Triangular Lattice Hubbard Model: A Density Matrix Renormalization Group Study, Phys. Rev. X 10, 021042 (2020).

[108] F. Ferrari and F. Becca, Dynamical Structure Factor of the $J_{1}-J_{2}$ Heisenberg Model on the Triangular Lattice: Magnons, Spinons, and Gauge Fields, Phys. Rev. X 9, 031026 (2019). 